\title{
Entre la liberación y la normalización. Representación de la disidencia sexual en Faustrecht der Freiheit de Rainer Werner Fassbinder
}

\author{
Atilio Raúl Rubino \\ IdIHCS / UNLP / CONICET \\ atiliorubino@yahoo.com.ar \\ Artículo bajo licencia Creative Commons \\ Atribución 4.0 Internacional (CC BY 4.0) \\ ENVIADO: 20-04-09 \\ ACEPTADO: 20-07-02
}

\section{RESUMEN}

Este artículo aborda el problema de la representación de la disidencia sexual en los años setenta, a partir de una película de Rainer Werner Fassbinder, Faustrecht der Freiheit. En el seno de los años setenta post-Stonewall y contemporáneo a los movimientos de liberación gaylésbica, el cine de Fassbinder refleja las tensiones propias de la época, entre la visibilización y la discreción, la conquista de derechos y la pérdida de libertades. El análisis de esta película permite ver cómo los debates que marcarían la emergencia de los movimientos queer de los noventa ya estaban presentes desde la década del setenta.

Palabras cLaVe

Disidencia sexual, Rainer Werner

Fassbinder, teoría queer, cine alemán.

\section{RESUMO}

Este artigo abrange o problema da representação da dissidência sexual na década dos setenta, baseado em um filme de Rainer Werner Fassbinder, Faustrecht der Freiheit. Em meados da década dos setenta, pós-Stonewall e contemporâneo aos movimentos de liberação gay-lésbica, o cinema de Fassbinder reflete as tensões típicas da época, entre a visibilidade e a discrição, a conquista de direitos e a perda de liberdades. A análise deste filme nos permite ver como os debates que marcariam o surgimento dos movimentos queer da década dos noventa, já estavam presentes desde a década dos anos setenta.

\section{Palavras-chave}

Dissidência sexual, Rainer Werner

Fassbinder, teoria queer, cinema alemão.

\section{ABSTRACT}

This article deals with the problem of the representation of sexual dissidence in the 1970s, based on a film by Rainer Werner Fassbinder, Faustrecht der Freiheit. In the heart of the post-Stonewall 1970 s and contemporary to the gay-lesbian liberation movements, Fassbinder's work reflects the tensions of the time, between visibility and discretion, the conquest of rights and the loss of freedoms. The analysis of this film allows us to see how the debates that marked the emergence of the queer movements of the 1990s had been already present since the 1970 s.

\section{KEYWORDS}

Sexual dissidence, Rainer Werner

Fassbinder, queer theory, german cinema. 


\section{LA LEY DEL MÁS FUERTE EN EL CONTEXTO DE LA LIBERACIÓN GAY-LÉSBICA}

Me gustaría empezar con un malentendido que resulta muy significativo. En 1975 Fassbinder realiza su primera película con un protagonista abierta y completamente gay (interpretado por él mismo), Faustrecht der Freiheit. El título se puede traducir como "la ley del más fuerte de la libertad". Para la avant premier en Estados Unidos, la película tuvo un título que era una traducción bastante fiel del alemán, Fistright of Freedom, pero no prosperó y, para su distribución internacional, se le cambió por el título por el que actualmente es conocida, Fox and his friends, porque la palabra "Fistright" era polémica, incómoda, debido a su connotación de fist-fucking (Stoneman, 1977: 42), una práctica sexual disidente, con la que no era conveniente asociar al cine gay de la época, más políticamente correcto, según los estándares de representación cinematográfica vigentes, sobre todo en Estados Unidos. ${ }^{1}$ El título de distribución internacional -en inglés- de la película borra la idea de pelea y lucha y la alusión a la libertad, que puede asociarse con los discursos liberacionistas de la década del setenta.

Por otra parte, la película de Rainer Werner Fassbinder Faustrecht der Freiheit (Fox and his friends, La ley del más fuerte) generó una recepción bastante negativa por parte, sobre todo, de la crítica gay del momento. Fue leída, por un lado, como un filme que presenta una visión trágica y negativa de la homosexualidad y que no genera solidaridad en la comunidad gay. ${ }^{2}$ Por otro, como un filme que no trata sobre la homosexualidad sino sobre las diferencias de clase. ${ }^{3}$ El filme ha decepcionado al público contemporáneo por estos dos motivos, porque no era leído como un filme sobre el tema de la homosexualidad, sino sobre la lucha de clases y la explotación de una clase social a otra y porque no da una visión positiva de la homosexualidad sino que presenta un retrato estereotipado de los personajes gay, más relacionado con la representación cinematográfica pre-Stonewall y alejado de las políticas gay de los setenta (Gregg, 2012: 564). Sin embargo, puede ser considerada hoy como una película que se adelanta a ciertos posicionamientos tanto del movimiento queer como de lo que se llamó el New queer cinema de los noventa, ${ }^{4}$ al correrse de la necesidad de representar positivamente a las minorías sexuales. De ahí la incomodidad que provocaba su cine en los setenta. Como una película provocadora pero lúcida, se puede pensar que advierte sobre la pérdida que significa que la comunidad gay se adapte a las normas de vida de la familia heterosexual, lo que hoy identificamos como homonorma. ${ }^{5}$

Según Woltersdorff, Fassbinder pone en escena la homosexualidad como un trasfondo para ampliar la perspectiva y vincularla con las luchas de clases, la cuestión de la edad, el racismo y demás. Las estrategias estéticas de Fassbinder se dirigen a cuestionar la idea de una minoría homosexual como un grupo homogéneo y distinto (Woltersdorff, 2015: 108). En su cine enfrenta la perspectiva identitaria propia de la década de la liberación gay-lésbica post-Stonewall a una más general que no implica el reconocimiento y aceptación de las minorías sexuales, sino la

Es importante mencionar que en esta época la sola visibilización del deseo homosexual era considerada pornográfica. Por eso, aún más rechazadas resultaban las prácticas disidentes como el fistfucking para el cine comercial.

2 Así lo describe Veit en su reseña de la recepción de la película de Fassbinder (1997: 116-129). Y, de hecho, Vito Russo no la menciona en su libro señero The Celulloid Closet (1981).

3 Bob Cant asegura "The fact that the main characters are gay men does of course make it interesting for gay men but it is not primarily a film which attempts to Deal With The Problem of Homosexuality" (Cant, 1976: 22). Andrew Britton también la critica porque no concierne a "the problem of homosexuality", concluyendo que la visión de la película sobre la homosexualidad "degrades us all, and should be roundly denounced” (1976: 16-7). Mildenberger (2001: 78) menciona también la pésima recepción que la película de Fassbinder tuvo en las cartas de lectores de la revista Du\&lch, así como la crítica aparecida en el número 9 de la revista $D O N$ de 1975. Asimismo, fue duramente criticado por los movimientos activistas, principalmente por el Homosexuelle Aktion Wetsberlin (HAW), entre otras cosas por la ausencia en su película de referencias a la Schwulenwebegung (Mildenberger, 2001: 80).

4 En esto coinciden críticos como Woltersdorff (2015) y Kuzniar (2000), entre otros.

Lisa Duggan retoma el término heteronormatividad de Warner (1993) para hablar también de la existencia de una homonormatividad a la que define como «a politics that does not contest dominant heteronormative assumptions and institutions, but upholds and sustains them, while promising the possibility of a demobilized gay constituency and a privatized, depoliticized gay culture anchored in domesticity and consumption». (Duggan, 2003: 50). 
crítica al capitalismo como sistema de producción de subjetividades y sexualidades normativas y binarias; es decir, la producción de humanidad y monstruosidad abyecta. En efecto, este artículo se propone analizar en particular en la película Faustrecht der Freiheit la perspectiva problematizadora de Fassbinder para pensar la representación de la homosexualidad. En el seno de los años setenta post-Stonewall y contemporáneo a la Schwulenwebegung -el movimiento de liberación gay de los setenta de la República Federal de Alemania- el cine de Fassbinder ya anticipa cuestiones que serán centrales en los cuestionamientos de la política sexo-disidente de fines de los años ochenta en adelante, la asimilación y normalización de ciertas identidades para generar nuevos sistemas de exclusiones y pertenencias. ${ }^{6}$ En ese sentido, este artículo aborda la problemática tensión entre sexo-disidencia y normalización sexual en la película de Fassbinder.

Franz Biberkopf -también conocido como Fox, por su apodo en el circo- pierde su trabajo porque arrestan a su jefe Klaus, con quien tiene una relación sexo-afectiva. Se dedica entonces a obtener sexo casual en estaciones y baños públicos, en donde conoce a Max, que lo introduce en la sociedad homosexual burguesa. Luego, Fox gana 500.000 marcos en la lotería, con lo que se convierte en presa fácil para homosexuales burgueses. Así conoce a Eugen y comienza una relación que lo va introduciendo en ese nuevo estilo de vida, al tiempo que éste se provecha de su nueva fortuna, para salvar las deudas de la empresa familiar, que estaba en ruinas, y para comprar un departamento elegante, decorarlo con muebles antiguos y cambiar el auto. Una vez que Eugen se aprovecha de todo el dinero de Franz, este ya no le sirve. Solo y errante, Franz muere en los pasillos del metro.

La película ha sido blanco de críticas negativas también porque combina las técnicas de distanciamiento brechtiano en boga en el cine-arte de la época con los elementos propios del melodrama hollywoodense a lo Douglas Sirk (Halle, 2012: 555 y Gregg, 2012: 564). Pero lo interesante es que el lugar de la heroína melodramática lo ocupa Fox, un homosexual de clase baja que de casualidad gana la lotería. Y es, justamente, el dinero -y no su sexualidadlo que desencadena la caída trágica. La película no retrata una salida del armario ni reacciones homofóbicas, sino que es la sociedad capitalista que convierte a las recientemente aceptadas sexualidades disidentes en una nueva norma atravesada por la clase social.7 Si la película fue criticada por tratar sobre la homosexualidad pero al mismo tiempo representarla de forma negativa, la clave está en representar una sociedad en donde la heternormatividad no es el único sistema de opresión, sino también la homonorma, el

6 Cabe aclarar que estos eran debates muy importantes en los movimientos sexo-disidentes tanto en Alemania como en Estados Unidos, ya que habían surgido en vinculación con movimientos de mujeres, con el Black Power y las luchas de izquierdas. Se puede mencionar como ejemplo el "Documento de trabajo para la convención constitucional revolucionaria" (1970) del Grupo de Liberación Gay de Chicago compilado por Mérida Jiménez (2009).

Es importante mencionar que en 1969 en la República Federal de Alemania se derogó parcialmente el artículo 175 del código penal que prohibía específicamente las relaciones homosexuales. Este hecho se dio de forma contemporánea a la rebelión de Stonewall en Estados Unidos y a la emergencia de los movimientos de liberación gay-lésbica a nivel transnacional. En Alemania a partir de 1971 comenzaron a organizarse las primeras agrupaciones de militancia que constituyeron la Schwulenbewegung. capitalismo que todo lo subsume a un bien de mercado. Ronald Gregg da una clave para pensar la particularidad de la película de Fassbinder. Si el tema es la lucha de clases, Fassbinder no convierte a la homosexualidad en un problema, como sí lo hacía el cine pre-Stonewall -pero también el cine gay de vanguardia de los setenta-, sino que plantea la sexualidad de los personajes como un trasfondo, un background, no como el tema central en el desarrollo de la trama (Gregg, 2012: 566). El propio Fassbinder explica en 1975:

It is certainly the first film in which the characters are homosexuals, without homosexuality being made into a problem. In films, plays or novels, if homosexuals appear, the homosexuality was the problem, or it was a comic turn. But here, homosexuality is quite different, it's a love story, where one person exploits the love of another person, and that's the story I always tell (Thomsen, 2004: 181).

Se podría decir que es este el tema central en casi toda su obra, la explotación o, más específicamente, las relaciones personales y amorosas pensadas como explotación/sumisión. Los protagonistas melodramáticos de Fassbinder están construidos sobre esta base, pero al mismo tiempo son los que generan empatía e identificación en el público. ${ }^{8}$ Por otro lado, es importante tener en cuenta que con este filme Fassbinder intentaba llegar a un público más amplio, él lo entendía como su primer filme comercial que permitiría consolidar un Hollywood alemán (Halle, 2012: 552), un cine narrativo comercial, rentable, internacional que compita con las producciones de Hollywood pero sin renunciar a la calidad. Se distanciaba, con esto, de buena parte del Neuer Deutscher Film, un cine de autor más independiente y experimental. De ahí la insistente utilización de las convenciones de los géneros cinematográficos masivos, como el melodrama. ${ }^{9}$

Según Richard Dyer, la película de Fassbinder no permite la identificación con los movimientos de liberación pero, sin embargo, puede provocar en la audiencia mayor discusión que un filme políticamente correcto (Dyer, 2002: 184). En este sentido, el cuestionamiento al modo de representación de la homosexualidad pasa por alto la ruptura en el cine de Fassbinder con cualquier idea de identidad como algo rígido, homogéneo y coherente. Por otro lado, la exigencia de representatividad privilegia una lectura en clave realista de la representación cinematográfica. Y, de hecho, en la recepción del filme también se puede notar una tensión entre una lectura realista o representacional y una en clave de melodrama. Refiere Halle (2012: 553) que en una entrevista de Joachim S. Hohmann en la revista Him, de agosto de 1975 , Fassbinder afirma que, si bien se desarrolla en un entorno gay, no es una película específicamente sobre las condiciones de la homosexualidad. 8 Como ejemplo se pueden mencionar películas como Angst essen
Seele auf (1974), que reescribe el melodrama de Douglas Sirk All That Heaven Allows (1955), In einem Jahr mit 13 Monden (1978), dedicada al igual que Faustrecht der Freiheit a su ex pareja Armin Meier, o Lola (1981), que reactualiza en clave de melodrama la película de Josef von Sternberg que la hizo famosa a Marlene Dietrich, Der blaue Engel (1930), basada en la novela Professor Unrat oder Das Ende eines Tyrannen (1905) de Heinrich Mann. Esta relación de explotación y sumisión también es una constante en su cine y aparece también en sus obras de teatro tempranas, como Tropfen auf heiße Steine (escrita alrededor de 1965/6) y Der Müll, die Stadt und der Tod (1975). Sobre estas últimas, cf. Rubino (2020).

Por eso, como comenta Halle, Rosa von Praunheim consideraba al cine de Fassbinder como una "kommerziellen Scheiß" (2012: 547) 
E insiste en que la 'realidad' de un filme está más en la recepción del público que en la película en sí misma. Así y todo, se enuncia como basada en una historia real.

Más que abonar a la explicación de la homosexualidad $-y$, con ello, inscribirse en un contexto de representaciones cinematográficas que definen qué es un homosexual-, la película de Fassbinder desnaturaliza los condicionamientos sociales que hacen que ciertas formas de vida se conviertan en identidades fijas susceptibles de ser definidas. Es decir, más que ocuparse de contribuir al conocimiento general acerca de qué es un homosexual, Fassbinder se ocupa de la homosexualidad, no como una condición personal, individual y esencialista, sino, por el contrario, como parte de un régimen político mayor, como parte de la producción de identidad, de normalidad y abyección, por parte de la heterosexualidad como régimen político del capitalismo.

\section{El fenómeno del CIRCo}

En una película que supuestamente no trata sobre la homosexualidad, es interesante ver cómo se representan diferentes ámbitos de socialización gay y las tensiones entre diferentes modos de vida. Es muy importante acentuar la marcada promiscuidad de Franz, que en la película no está vista con signo negativo. Sus levantes ${ }^{10}$ en baños públicos y lugares de paso y de cruising, las demostraciones de afecto públicas con Klaus, entre otras prácticas, aparecen visibilizadas de forma positiva. A diferencia de películas posteriores como Philadelphia (1993), la promiscuidad no es la causante de las desgracias del protagonista. ${ }^{11}$ En Faustrecht der Freiheit la promiscuidad no tiene un signo negativo ni es la causante de las desgracias del protagonista, no es lo que lo lleva a la ruina.

Es interesante pensar, en ese sentido, el inicio de la película. Klaus como animador del circo, se encarga de presentar a todos los artistas mientras dos policías que han ido a arrestarlo esperan en el auto. El momento en el que deciden llevar adelante el arresto es cuando Klaus presenta a la atracción más importante, Fox der sprechende Kopf. Ante el alboroto, Fox sale de atrás del telón y despide a Klaus, que está siendo llevado por la policía, con un beso en la boca. Este beso al inicio de la película, sienta las bases para la presencia de la homosexualidad como background y no como un problema. A su vez, es interesante tener en cuenta que la puesta en escena presenta esta situación arriba del escenario, en medio de la presentación, como si se tratara de una representación dentro de la representación. Es, en este sentido, una exhibición muy marcada en la puesta en escena. Fox, como schwul-palabra alemana que designa la identidad marica-, como clase obrera, es un freak, un monstruo (esa es la palabra con la que lo define Philip cuando lo ve cenando con Eugen). Monstruo, freak, queer, disidente, el margen de lo socialmente aceptado, lo no completamente humano, el borde de la humanidad. ¿Por qué Fox der sprechende Kopf es la máxima atracción del circo? ¿Se trata de la homosexualidad como exhibicionismo voyeurista? ¿Es el fenómeno de circo exhibido para el ojo voyeur? Si como atracción principal Fox es el monstruo del freak-show, se trata entonces de una forma de designar algo no humano ni animal, algo al borde de la humanidad.

Quizá ese tipo de representación es de la que Fassbinder intenta alejarse, distanciarse. O quizá quiere generar reflexión sobre la representación como posibilidad, sobre la relación del cine con lo real. Cuando Franz ve que están arrestando a Klaus, sale y lo despide con un beso en la boca y con un abrazo y una demostración de afecto disidente muy marcada. Al modo de la resignificación que ocurriera en los noventa con el término queer, podríamos pensar

\footnotetext{
10 Se utiliza en este artículo la expresión en español rioplatense "levante" para referirse a lo que en España se llama "ligue" o en inglés "cruising". Se trata de encuentros sexuales casuales en lugares públicos o entre personas que se conocen en lugares públicos de forma casual.

11 Para considerar la importancia de este tipo de representaciones con signo positivo, hay que pensar en lo que implica, por ejemplo, la promiscuidad de los espacios de sociabilización y sexo casual gay para una película mainstream de los años noventa como Philadelphia (Jonathan Demme, 1993). En el juicio final de la película se usa como argumento en contra de Andrew Beckett (Tom Hanks) que él ha tenido sexo casual en un cine porno, con el agravante de que constituye una infidelidad porque estaba en pareja con Miguel Alvarez (Antonio Banderas). Lo hizo una sola vez, pero eso basta para ser culpable de su enfermedad. Su promiscuidad, aunque mínima y vergonzante, es lo que lo condena moralmente.
} 
que, al besar a Klaus en la boca, Fox exhibe su monstruosidad, la visibiliza y, con eso, la resignifica con signo positivo, la muestra con orgullo. Al mismo tiempo, al interpretar al personaje el propio director y ponerle el cuerpo, constituye también la exhibición orgullosa de su propia salida del armario, como lo sugiere LaValley (1994). Franz se vuelve agente de su sexualidad. Y se trata de una agencia de visibilización política. La exhibición circense del freak se convierte en una visibilización reivindicatoria y orgullosa, en una resignificación queer. Pero ese gesto, que podemos pensar como queer (avant la lettre, obviamente), está al inicio de la película, ofrece solo el marco, para narrar la vida de un personaje melodramático. De hecho, se podría pensar a la película a partir del eje orgullo/vergüenza o visibilización/discreción. De esta forma, la película deja de lado la narrativa del coming-out. ${ }^{12}$ Todos los personajes están fuera del armario. Pero no de la misma manera o en el mismo grado, porque el eje de la sexualidad hay que cruzarlo con el de clase o, incluso, con el de raza.

La película exhibe abiertamente la sexualidad promiscua. Se visibilizan prácticas como el cruising en las calles, se reproducen los rituales de levantes, las miradas, los espacios. Lejos de la representación negativa, los lugares en donde se llevan a cabo prácticas promiscuas, como baños y saunas, devienen, en cierta medida, utópicos o ideales, ya que en ellos se suspenden las diferencias de clases $u$ otros condicionamientos sociales y queda en primer plano únicamente el placer. De hecho, Fox conoce primero a Max en un baño público. El contacto sexual entre ambos constituye, asimismo, un encuentro casual interclase, imposible de llevarse a cabo en otro contexto o lugar de socialización en donde las relaciones de clase estarían en primer plano. De esta forma, el inicio de la película pone en escena el espacio del baño que puede leerse como utópico, en donde lo único que importa es el sexo, y no la mirada social.

Cuando lo lleva a su casa en el auto, Fox decide detenerse para comprar el billete de lotería que lo haría rico, pero logra que se lo vendan solo cuando intercede Max, con su apariencia de clase y modales más refinados. Este detalle menor marca, justamente, hasta qué punto hay una concepción utópica de lo sexual, como puro disfrute, como producción de placeres apartados de la mirada social y de la generación de vidas más valiosas que otras.

Mediante una elipsis la película nos muestra luego la casa de Max. Fox ya ganó la lotería, por lo que ahora ya no es visto como antes 0 , mejor dicho, su vida ya no vale tan poco como antes, pues vale por su dinero. Allí también conoce a quien será su pareja, de quien se enamorará y cuya relación lo llevará a la ruina, Eugen. La puesta en escena muestra de un lado a Franz, solo, como si estuviera en un escenario, exhibido nuevamente como un fenómeno de circo. Del otro lado, a Max, Eugen y su pareja Philip que lo

12 El coming-out o salida del armario designa el hecho de contar abiertamente y hacer pública la orientación sexual. Claro que esta expresión en un principio se usa solo en el caso de gays y lesbianas. La heterosexualidad no tiene una salida del armario, porque se da por sentada, es parte de un régimen político que heterosexualiza compulsivamente a todos los sujetos. En el cine, suele hablarse de "narrativa de coming-out" para referirse a aquellas películas de temática homosexual que narran cómo los personajes descubren su sexualidad y la hacen pública de determinada manera. Esta temática es la que ha hegemonizado al cine gay-lésbico por mucho tiempo. Se trata de un juego en torno a la visibilidad e invisibilidad, a la idea del secreto y la confesión que Sedgwick (1998) analiza como una "epistemología del armario". miran, observan su apariencia y comportamiento de clase baja y comentan maliciosamente, como si fueran espectadores de un Freak Show.

Después de Max, Eugen lo llevará a su casa. Pero las intenciones de placer sexual de Fox no son las mismas que las de Eugen. Fox ya ha ganado la lotería. Frente a la libidinización de la carne y del sexo por parte de Fox, tenemos la libidinización del dinero por parte de Eugen. Lo que quiere es su dinero. Con un gesto marcadamente melodramático la película ubica de forma maniquea a Eugen en el lugar del villano. En este primer encuentro sexual a partir del cual comienzan su relación, también Fox muestra su promiscuidad, pues inmediatamente se desnuda y le pregunta por la cama. A la mañana siguiente se da la primera escena de ocultamiento. Cuando llega Philip, Eugen hace esconder a Franz en el baño. Es lo que marca que se trata de una relación clandestina, oculta, lo que contrasta con la promiscua vida sexual activa y desinhibida de Franz. Philip huele los pantalones que apestan a baños públicos. Opera aquí un nuevo cuestionamiento a este tipo de vida sexual. Pero se trata de un cuestionamiento de clase dirigido a la puesta en práctica de una sexualidad activa y sin vergüenza. Eugen lo enuncia como "un pequeño desliz".

Lo que aparece remarcado es la hipocresía respecto a ese tipo de vida, se trata siempre de guardar las apariencias. En contraste con el beso que se dan Fox y Klaus al inicio de la película, es importante remarcar que cuando Eugen lo va a buscar al bar gay lo saluda con un beso en la mejilla, no en la boca. El bar, por su parte, tiene un carácter de apertura y visibilidad muy marcado. Las demostraciones de afecto sexual son desinhibidas y abiertas. Por ejemplo, una travesti entra en el baño "Für Herren". Luego, cuando sale, besa en la boca a Peter, uno de los amigos del bar de Fox. Este beso contrasta con la demostración de afecto discreta entre Fox y Eugen. A Eugen obviamente le da asco el bar y se niega a comer o tomar algo allí y lo lleva a un restaurante elegante. Se trata, evidentemente, de una vergüenza de clase social, porque lo que a Eugen lo abochorna son las señales que evidencian la clase social a la que pertenece Fox y el modo en el que no puede encajar en su mundo de clase burguesa acomodada. Pero también podríamos pensar qué relación guarda con la discreción o la visibilidad de la disidencia sexual. En la que el beso marca la diferencia. El restaurante al que lo lleva Eugen es heterosexual, de gente grande, de clase alta. La carta está en francés. Y Fox lo avergüenza cuando hace un truco con las manos y la gente comienza a mirarlos. Eugen le pide que deje de hacerlo porque los están mirando. Opera aquí una torsión y conversión de la vergüenza gay en vergüenza de clase social.

La película se puede leer en las minuciosidades de la salida del armario, pues no plantea estrictamente una narrativa de coming-out, pero sí se puede pensar a partir de estas oposiciones orgullo/vergüenza y visibilización/ discreción. Al conectar sexualidad con capitalismo, el cine de Fassbinder ya da cuenta de esa normalización de lo gay que será conceptualizada luego como una de las crisis del movimiento gay-lésbico a mediados de los ochenta que produce posteriormente el surgimiento de la perspectiva queer. No se trata de que el tema de la sexualidad sea irrelevante, sin importancia, sino que se lo trabaja a partir de esta vinculación. 


\section{CUERPOS PORNOGRÁFICOS Y MERCADO}

Asimismo, es importante mencionar la exhibición de cuerpos masculinos como objeto de deseo y placer homosexual, que puede pensarse en vinculación con los cuerpos de la pornografía gay de los setenta como visibilización política de la disidencia, pero también como producción de cuerpos estereotipados. La pornografía gay audiovisual de los años setenta tenía un importante contenido político en el sentido de visibilización del placer homosexual y de su objeto de deseo en cuerpos masculinos exhibidos para el ojo gay. ${ }^{13}$ De esta forma, en el cine de Fassbinder se produce una inversión del "male gaze" propio del cine de Hollywood (Mulvey, 1999) que conceptualizara la teoría feminista del cine. Con el impacto de la liberación gay durante los setenta sus películas muestran un uso gay explícito de los cuerpos masculinos. Podemos pensar, a modo de ejemplo, la escena en la que Fox y Max se encuentran en la sauna. Cuando Max está allí, metido en el barro, pasan dos chicos desnudos. Luego, su primer plano está enmarcado por un desnudo masculino y por la belleza del pene. Se trata, por un lado, de cuerpos hegemónicos, haciendo alarde de sus penes y, por otro, la puesta en escena y el encuadre los exhibe sin cabeza, anónimos "as if they were interchangeable for entry into this world" (LaValley, 1994: 127). Asimismo, son muchas las alusiones al pene y su tamaño durante toda la película, como modo de visibilizar el deseo sexual. Así, por ejemplo, cuando Max presenta a Fox ante Eugen y Philip, estos solo atinan a preguntar por el tamaño de su pene, desde el prejuicio de no encontrarle otro posible atractivo. Asimismo, cuando Eugen es sorprendido por Philip con Fox después de la primera noche juntos, este le dice que solo fue un pequeño desliz y Philip pregunta irónico "wie klein? zwanzig Zentimeter? oder achtzehn? oder noch kleiner?” [¿Cuán pequeño? ¿De 20 centímetros? ¿O de 18? ¿O incluso más pequeño?]. También en Marruecos Fox insiste una y otra vez en querer conocer el tamaño del pene de Salem. La representación de la desnudez y la insistencia en el pene y su tamaño juegan un papel de visibilización política de la sexualidad similar al de la pornografía audiovisual de los setenta (LaValley, 1994: 115). Pero, al mismo tiempo, se puede decir que marca la normativización de los cuerpos, cuáles son cuerpos aceptables y cuáles no para el deseo homosexual. El porno como tecnología no solo visibiliza prácticas y deseos disidentes sino que también institucionaliza cuáles cuerpos son deseables y cuáles no, así como qué prácticas son placenteras, mediante la instauración de cierta retórica del acto sexual. En ese contexto, el cuerpo de Fassbinder no se adapta a las normas, es un cuerpo gordo, feo, viejo (eso le dice Max, por ejemplo, cuando lo ve desnudo y también el vendedor de flores que, hacia el final de la película, quiere tener algo con él porque le gustan los feos). Este detalle es el que también permite avizorar una incipiente normalidad gay. Fassbinder pone el modelo de cuerpo gay deseable en la escena de la sauna en contraste con la desnudez del suyo propio. Wayne Koestenbaum (2012) menciona la importancia de que el propio director muestre su pene, debido a que Fassbinder era considerado un hombre feo, según los estándares de belleza gay de la época. Para Koestenbaum, entonces, el

13 Resulta importante mencionar que el primer cine porno gay tenía un importante carácter político de visibilización y liberación de la sexualidad. En una película porno de 1972, L.A. Plays Itself (dir. Fred Halsted), por ejemplo, podemos ver planos de la ciudad de Los Ángeles, con graffitis que dicen "Gay Power". desnudo frontal de Fassbinder “isn't just a nude scene. It's a pride scene" (Koestenbaum, 2012: 69).

A su vez, esta normativa para el deseo sexual gay se vincula con un incipiente mercado. Esto se puede ver claramente en la escena en la agencia de turismo cuando sacan el pasaje para Marruecos. La vendedora ya conoce a Eugen y le ofrece alternativas para turismo gay, lugares en donde pueden conseguir sexo casual con hombres del lugar e, incluso, insinúa la presencia de taxi-boys como parte del atractivo turístico. Así deciden ir a Marruecos, no sin la sorpresa de la agente de turismo cuando se da cuenta de que el que paga el viaje no es Eugen sino Fox. Una vez allí, van a un punto de cruising, de levante, donde intentan un encuentro con Salem (interpretado por El Hedi ben Salem). Esta escena está marcada por la retórica de un levante casual gay en un espacio público. Sin embargo, cuando van al hotel, no los dejan entrar con Salem porque no pueden ingresar árabes y el conserje les ofrece, en cambio, llamarles un garçon. El cuerpo de Salem es también uno que no encaja. Aquí es cuando la cuestión sexual se cruza no solo con la clase sino también con la raza. Y, al mismo tiempo, se vincula con esa institucionalización incipiente de un mercado gay. Esa es, justamente, la particularidad del cine de Fassbinder y de esta película. Si bien las relaciones entre género, sexo, clase social, raza, edad, etc. ya estaban siendo discutidas en los movimientos gay y lésbicos desde mediados de los setenta (Mérida Jiménez, 2009: 39), en Faustrecht der Freiheit se vislumbra al capitalismo no sólo como un gran sistema de opresiones múltiples, sino también con con enorme poder asimilador. La prostitución masculina es uno de los atractivos turísticos de Marruecos pero sólo entre personas blancas y, claramente, de clase alta.

En el cine de Fassbinder, la familia nuclear burguesa es un modelo que resulta perverso. Por eso, la normalización de las relaciones homosexuales bajo el modelo de la familia heterosexual aparece tensionado con otras posibles formas de vinculación sexo-afectiva no monogámicas. Si la homosexualidad después de 1969 ya no está penada y es aceptada, es necesario tener en cuenta que lo que prevalece como único modelo es la familia nuclear (Halle, 2012: 560). Pensar esta visión fassbindereana desde el punto de vista individual, personal o de minorías es lo que nos da por resultado considerarlo como un director que ofrece perspectivas negativas sobre la homosexualidad que no permiten ningún tipo de liberación o resistencia. Más interesante es pensar desde una perspectiva foucaultiana que lo que Fassbinder está intentando retratar es el funcionamiento de un sistema. Si sus personajes son opresores y oprimidos al mismo tiempo, sádicos y masoquistas, explotadores y explotados, es porque no hay un afuera del poder y, justamente por eso, la posibilidad de resistencia se encuentra en el hecho de ser parte de ese sistema.

La familia gay puede ser igualmente patriarcal que la heterosexual, como lo demuestra la relación con Eugen, quien -más allá de su sexualidad- es también un patriarca como su padre. Es interesante pensar aquí en un detalle menor. Cuando Fox ve la foto del padre de Eugen en un portarretratos expresa sentirse atraído sexualmente. Esto es algo que Eugen no puede comprender, porque para él es no solamente su padre, sino la figura del patriarca familiar. El retrato de su padre es la celebración del patriarcado 
capitalista. Se produce un contraste de la figura del padre como patriarca dueño de la empresa familiar, por un lado, y como simplemente un hombre, pasible de ser un objeto de deseo schwul, por otro. Para Eugen es su modelo a seguir en la empresa familiar, para convertirse él también en un patriarca. El deseo de Fox, en este sentido, también rompe con las limitaciones de edad. La reacción de Fox opera como un desvío, enunciando su deseo sexual hacia el padre de la persona con la que se está por acostar.

Por otro lado es importante preguntarse por las diferencias entre la relación que tenía con Klaus y la que ahora tiene con Eugen, así como la de Eugen y Philip. Claramente estas últimas son relaciones burguesas normales ¿Pero se trata solo de una cuestión de clase? ¿Qué ocurre con la reivindicación del sexo y la promiscuidad? A partir de ciertos indicios que da la película podemos preguntarnos si la relación con Klaus era monogámica, como la que tiene con Eugen, o la que tenían Eugen y Phillip. Cuando Klaus sale de la cárcel se encuentra en el bar gay y se saludan afectuosamente. Nada parece indicar que la relación de Fox y Klaus sea una relación de pareja, sino que tranquilamente podría tratarse de otro tipo de vínculo sexo-afectivo, no normalizado, no institucionalizado, probablemente una relación no exclusiva. Diferente a la relación de noviazgo que tiene con Eugen. Este, cuando van a la boutique, se besa a escondidas con Phillip, se ocultan. De modo que constituye un claro engaño a Fox, porque mantiene clandestinamente una relación con su ex que constituye un acto de infidelidad, solo posible en una relación monogámica. De esta forma, se pone en primer plano la hipocresía de las relaciones sexo-afectivas institucionalizadas en la pareja que sigue los lineamientos de la familia nuclear heterosexual. Por otro lado, se puede pensar en cierta endogamia en los vínculos de la clase alta gay: la tienda de antigüedades en la que amueblan el nuevo departamento es de Max, la boutique en donde lo viste y le cambian el aspecto a Fox pertenece a Phillip. Hasta dónde esa vergüenza de clase social se puede pensar como una norma sobre cómo se debe vivir la sexualidad, sobre la forma correcta de ser homosexual y ser aceptado por la sociedad. Si bien es verdad que las representaciones de los ambientes de socialización y de los personajes gay son un poco estereotipadas, también es cierto que implican una enorme visibilización.

\section{EL FIST-FUCKING DE LA LIBERTAD}

Quisiera volver nuevamente al malentendido planteado al inicio para pensar, justamente, qué tipo de discreción opera en el hecho de que el título no pueda connotar una práctica sexual. El discurso del porno gay en los setenta (así como el del porno heterosexual) tenía también una importante dimensión política, que implicaba la liberación de la sexualidad y la visibilización de prácticas disidentes. A su vez, el cine pornográfico gay a mediados de los años setenta "promulgated the erotic promise of the sexual revolution: sex without apology, without restraint, and without distinction." (Escoffier, 2009: 114). La retórica del porno todavía no estaba completamente institucionalizada, pero sí comenzaba a existir una formalización de cuáles eran los cuerpos aceptables y cuáles no. Esta doble dimensión la encontramos en las relaciones con la pornografía en la película de Fassbinder. La sexualidad promiscua en los baños y en las saunas se muestra sin ningún registro negativo, forman parte de los logros de la liberación gay-lésbica de los setenta, pero al mismo tiempo, no todos los cuerpos tienen el mismo valor. Los jóvenes que se pasean desnudos cuando Franz y Max se encuentran en la sauna, son cuerpos pornificables, implican el modelo de belleza gay, están puestos ahí no para el placer visual del ojo heterosexual, como ocurría con casi todo el cine mainstream de Hollywood, sino para el placer visual del ojo gay. Pero al mismo tiempo constituyen una estandarización y normalización de la belleza. Según esos cánones el cuerpo de Fassbinder no es aceptable, por eso no deja de exhibirlo en su desnudez y de marcar que no encaja en los modelos de belleza. Le dicen que es feo y también que está gordo. Es la grieta que se abre en la liberación homosexual de los setenta. Si el porno implica liberación, poco a poco, como habrá de notarlo décadas más tarde Preciado (2014), también implica adoctrinamiento y disciplinamiento. El porno enseña cuáles son las prácticas correctas y cuáles no, cuáles los cuerpos aceptables como deseables y cuáles no. El porno es un dispositivo biopolítico. Y esto ya comienza a verse, muy levemente, en el uso que Fassbinder hace de sus retóricas.

Si el cine gay de los setenta exigía que haya representaciones positivas de la disidencia sexual, recién, según la crítica, con el New Queer Cinema norteamericano, ${ }^{14}$ se va a festejar el planteo de otras problemáticas. El cine anterior estaba muy asociado a las políticas identitarias del movimiento gay-lésbico. En paralelo al surgimiento del movimiento queer en los noventa, el cine comienza a mostrar nuevas direcciones para las representaciones de las sexualidades disidentes. Pero según Mennel (2012), el New Queer Cinema sigue la línea de la tradición de cineastas queer de la vanguardia como Andy Warhol, Rainer Werner Fassbinder, Keneth Anger y John Waters (Mennel, 2012: 67). Lo que la crítica vio de rupturista y novedoso en el New Queer Cinema ya ocurría en el cine europeo de los años setenta.

14 El término New Queer Cinema fue acuñado por Ruby Rich (1992) en un ensayo muy influyente, en donde él notaba que en los años 1990-1992 se estaban produciendo cambios en las representaciones de la disidencia sexual en el cine norteamericano, principalmente a partir de ciertas películas muy vistas en los Festivales de Toronto y Amsterdam. Entre los directores más destacados se encuentran Gus Van Sant, Todd Haynes, Gregg Araki, Derek Jarman y Bruce LaBruce, entre otros. 
En este sentido, me interesa pensar dos cuestiones, en primer lugar hasta qué punto Fassbinder se adelanta a la normalización de lo gay y a la instauración de un mercado gay, es decir, de una adaptación de las vidas disidentes a las normas de la familia burguesa, que el cineasta considera como imperativos perversos. Por otro lado, por qué se leyó a Faustrecht der Freiheit como una visión negativa y pesimista de la sexualidad cuando, en realidad, está manifestando una representación positiva de lo que la homosexualidad tenía de más disidente: la promiscuidad y el cuestionamiento al statu-quo, la búsqueda o producción de nuevas formas de placer y de nuevas formas de relacionarse (Foucault, 2016: 91-2). En este sentido, la confrontación con el statu-quo y la institución de la familia burguesa es también un cuestionamiento al confort de la liberación gay (LaValley, 1994: 117). Quizá sea posible pensar que estas críticas que se le hacían a Fassbinder tenían un carácter un poco conservador, quizá verían con mejores ojos el aleccionamiento acerca de los riesgos de la promiscuidad y de la importancia de constituir una familia, sea cual sea la orientación sexual que se tenga. Es decir, que las personas homosexuales también podían encontrar el amor-verdadero, trascendental-y llevar adelante una vida decente, es decir, en familia monogámica. El cuestionamiento a la decencia burguesa como un valor de clase que atentaba contra los verdaderos logros de la liberación homosexual no podía ser leído con claridad todavía en los setenta. Quizá hoy sí, después del auge de los estudios queer, puede ser mejor dilucidado. Quizá, como comenta Rentschler, "Fassbinder embodied the hope, irritation, and disenchantment of his generation" (Rentschler, 2012: 424).

Si a partir de los noventa el giro queer ha logrado ligar a la sexualidad con otros temas y no pensarla como compartimentos estanco, relacionándola con género, raza, clase, discapacidad, etc; por qué no se puede pensar que esto ya estaba siendo planteado en los setenta. Y, en este sentido, quizá sí podamos ver un atisbo de agenciamiento en el cine de Fassbinder. Es verdad que el final del personaje es trágico, pues muere solo en la estación del metro. Klaus y Max pasan, lo miran y lo dejan tirado cuando ven que está muerto. Los niños juegan con el cadáver y le roban la billetera todo un signo de que, una vez despojado de su dinero, ya no es una vida, ya no importa, no interesa, es un no-humano. Para Richard Dyer la relación entre sexualidad y clase en Fassbinder resulta ambigua, y esto se debe a lo que él llama "left-wing melancholy", lo cual implica "a view of life that recognises the exploitativeness of capitalist society but is unable to see any means by which a fundamental change in this society can take place" (Dyer, 2002: 176). Desde esta perspectiva las clases oprimidas, trabajadores, mujeres y gays, son víctimas sin agencia y, en ese sentido, cómplices de su explotación:

"the emphasis in Fassbinder's films on people as victims of society and history implies a model of social change that is unable to indicate how people can be actively involved in that process of change; it implies, in other words, a model that makes political struggle pointless" (Dyer, 2002: 181).

Así se ha interpretado generalmente la muerte del personaje, por eso es una película pesimista, que no ofrece una salida al sistema capitalista de opresión. Sin embargo, creo que en ciertos gestos del personaje se puede avizorar otra perspectiva. Es interesante revisar, al respecto, lo que ocurre justo antes de su muerte, después de haberse quedado sin dinero por el engaño de Eugen. Fox intenta dos veces tener sexo con unos soldados norteamericanos. En la primera ocasión, en la puerta del bar, no se entienden porque no hablan el mismo idioma y ellos lo que buscan es tener relaciones sexuales con chicas. Pero en el segundo, ya dentro del bar, ellos comprenden y, si bien parecen ser heterosexuales, aceptan tener sexo con Fox, pero solo por dinero. "How much do you pay?", le preguntan. Y Fox comienza a recorrer el bar, como perdido y repitiendo "Was ist zahlen? Er will wissen, was ist zahlen? ¡Ich zahle alles! ¡Ich zahle immer alles!. ¡Ich muss alles zahlen! ¡Immer! [¿Cuánto pago? Quiere saber cuánto pago. ¡Lo pago todo! ¡Siempre pago todo! ¡Tengo que pagar todo! ¡Siempre!]. Esta respuesta puede pensarse como una conciencia melodramática por parte del personaje que, a su vez, adelanta su trágico final. Es lo que él tiene que pagar por ser quien es, por ser una existencia marginal, que no cuenta como humana para el sistema capitalista, que se puede dejar morir. Aparece aquí también, podríamos pensar, el masoquismo del personaje como una agencia (Deleuze y Guattari, 1988). Elige ser víctima antes que formar parte de un sistema perverso. Y cuya perversidad nada tiene que ver con la sexualidad. O sí, quizá, con la sublimación de la sexualidad y la libidinización del dinero y el status. Con el ocultamiento de la sexualidad, con el hecho de retrotaerla al espacio privado guardando, en lo público, la discreción.

A modo de conclusión, se puede decir que Fassbinder se adelanta a ciertas cuestiones que llegarían al cine y también a los debates públicos mucho después. Si bien las películas de Fassbinder nunca han creado el entorno de nuevos movimientos sociales ni influido en las agendas políticas de identidad de la Frauenbewegung, la Schwulenbewegung y la cultura Gastarbeiter (Tedjasukmana, 2015: 47), la distancia crítica del realizador puede ser leída hoy en día como anticipando algunas de las cuestiones que ya en los años noventa sí aparecerían en los debates públicos sobre sexualidades. Pero eran tensiones que ya estaban presentes en la década del setenta y que se pueden ver en la estructura del sentir que plantean estos textos culturales.

Me interesa marcar también en este caso cómo diferentes prácticas y diferentes perspectivas convivían en los setenta, pero también diversas formas de sentir y experimentar la sexualidad. La despenalización de la homosexualidad y los avances del movimiento de liberación eran vividos también por un sector como una pérdida de libertades sexuales porque implicaba una incipiente normalización de las vidas homosexuales, una segmentarización de los puntos de fuga que significaba la liberación. La película de Fassbinder, en este sentido, permite ver cómo mediando los años setenta ya se estaba en presencia de perspectivas sexo-disidentes, incluso respecto a la política identitaria de la homosexualidad, que ya adelantan, de alguna manera, lo que posteriormente sería conceptualizado como normalización de lo gay o, más recientemente, como homonorma. 
Britton, A. (1976). Foxed: A Critique of "Fox". Gay Left 3 (1976), 16-17.

CANT, B. (1976). Fassbinder's Fox. Gay Left 2 (1976).

Deleuze, G.; Guattarı, F. (1988). Mil mesetas: capitalismo y esquizofrenia. Valencia: Pre-textos.

Duggan, L. (2003). The twilight of equality? Neoliberalism, cultural politics, and the attack on democracy. Boston: Beacon Press.

DYER, R. (2002). The Culture of Queers. London: Routledge.

EsCoffIER, J. (2009). Bigger than Life. The History of Gay Porn Cinema from Beefcake to Hardcore. Filadelfia: Running Press.

FASSBINDER, R. W. (2002). La anarquía de la imaginación: entrevistas, ensayos y notas. Barcelona, Buenos Aires, México: Paidós.

Foucault, M. (2016). De la amistad como forma de vida. En: Escritos y entrevistas 1978-1984. Buenos Aires: El cuenco de plata, 87-94.

GregG, R. (2012). Fassbinder's Fox and His Friends and Gay Film as Counter-Public. En Peucker, B., ed. A companion to Rainer Werner Fassbinder. Chichester, West Sussex, Malden, MA: Wiley-Blackwell, 564-578.

Halle, R. (2012). Rainer, Rosa, and Werner: New Gay Film as Counter-Public. En Peucker, B., ed. A companion to Rainer Werner Fassbinder. Chichester, West Sussex, Malden, MA: Wiley-Blackwell, 542-563.

HALPERIN, D. (2007). San Foucault: para una hagiografía gay. Buenos Aires: El cuenco de plata.

Koestenbaum, W. (2012). Five Fassbinder Scenes. En Peucker, B., ed. A companion to Rainer Werner Fassbinder. Chichester, West Sussex, Malden, MA: WileyBlackwell, 67-76.

KuZnIAR, A. (2000). The Queer German Cinema. Stanford, Stanford. California: Stanford University Press.

LAVALLeY, A. (1994). The Gay Liberation of RainerWerner Fassbinder: Male Subjectivity, Male Bodies, Male Lovers. New German Critique, No. 63, 108-137.

Mennel, B. (2012). Queer Cinema. Schoolgirls, Vampires, and Gay Cowboys. New York: Wallflower Press.

MÉRIDA JIMÉNEZ, R., ED. (2009). Manifiestos gays, lesbianos y queer. Testimonios de una lucha (1969-1994). Barcelona: Icaria editorial

Mildenberger, F. (2001). Klassenkampf auf der Leinwand - Grabenkrieg in der Diskussion. Zur Rezeption von Rainer Werner Fassbinder Film Faustrecht der Freihet. FORUM: Homosexualität und Literatur, № 38, 77-83.

Mulvey, L. (1999). Visual Pleasure and Narrative Cinema. En Braudy, L.; Cohen, M., eds. Film Theory and Criticism. Introductory Readings. New York: Oxford University Press, 833-844.

Preciado, P. B. (2014). Testo yonqui: sexo, drogas y biopolítica. Buenos Aires: Paidós.

RentSChleR, E. (2012). “There Are Many Ways to Fight a Battle”: Young Fassbinder and the Myths of 1968. En Peucker, B., ed. A companion to Rainer Werner Fassbinder. Chichester, West Sussex, Malden, MA: Wiley-Blackwell, 423440. 
Rubino, A. (2020). El fist-fucking de la libertad. La liberación sexual en dos textos teatrales de Rainer Werner Fassbinder. Anagnórisis. Revista de investigación teatral, Núm. 22, pp. 88-110

Sedgwick, E. (1998). Epistemología del armario. Barcelona: Ediciones de la Tempestad.

SAXE, F. (2013). Representación transnacional de las sexualidades disidentes en textos culturales alemanes recientes (1987-2012). Tesis de Posgrado, Doctorado en Letras, FaHCE-UNLP.

Stoneman, E. D. (1977). The Evolution of a Filmmaker. Blueboy, XV (December 1977).

Tedjasukmana, Ch. (2015). Negative Solidarität. Zur Aktualität Rainer Werner Fassbinders. Text + Kritik, $N^{\circ} 103,44-53$.

Thomsen, Сн. (2004). Fassbinder. Life and Work of a Provocative Genius. Minneapolis: University of Minnesota Press.

VEIT, R. (1997). Wie die Schwulen ins Kino kamen. Ein Rückblick mit schnellem Vorlauf. En Kraushaar, E., ed. Hundert Jahre schwul. Eine Revue. Berlin: Rowohlt, 116-129.

WARNER, M. (1993). Fear of a queer planet. Queer politics and social theory. Minneapolis: University of Minnesota Press.

WoltersdorfF, V. (2015). "Grundsätzlich ist jeder homosexuell, und deshalb ist es kein Problem". Homosexuelle Minderheiten und Fassbinders Filme Text + Kritik, $\mathrm{N}^{\circ} 103,108-120$. 